\title{
Prediction and prevention of sudden cardiac arrest
}

\author{
Heikki V. Huikuri MD
}

See related research article by Reinier and colleagues on page 1705 and at www.cmaj.ca/lookup/doi/10.1503/cmaj.101512

Competing interests: None declared.

This article was solicited and has not been peer reviewed.

Correspondence to: Dr. Heikki V. Huikuri, heikki.huikuri@oulu.fi

CMAJ 2011. DOI:10.1503 /cmaj.111245

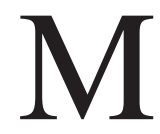
ajor advances have been made in understanding the causes of and treatments for cardiovascular disease, and mortality related to such disease has been reduced. However, the incidence of sudden cardiac arrest has remained almost unchanged for decades, generally affecting younger people (mean age about $65 \mathrm{yr}$ ) more so than other cardiovascular conditions causing death. ${ }^{1}$ Sudden cardiac arrest therefore represents a heavy burden to families, communities and the health care system.

Several reasons have been identified for the lack of improvement in outcomes for people at risk of premature, unexpected, sudden cardiac arrest, such as insufficient understanding of the mechanisms responsible or of the role played by genetic or environmental factors and the lack of good parameters for stratifying risk. The study by Reinier and colleagues published in the CMAJ has tested the hypothesis that environmental factors, such as those found in North American neighbourhoods with lower socioeconomic status, are associated with a higher incidence of sudden cardiac arrest. ${ }^{2}$

The high incidence of cardiac arrest is recognized as the predominant mechanism of sudden cardiac death, especially among patients with coronary artery disease. As such, medical scientists and clinicians have sought ways of predicting and preventing these events. Previously identified variables that help predict the risk of sudden cardiac arrest among patients with structural heart disease include the left ventricular ejection fraction, various markers of cardiovascular autonomic function, electrical markers measured from standard and exercise electrocardiograms and biomarkers such as plasma levels of natriuretic peptides and markers of inflammation. ${ }^{3}$

Despite the multiplicity of factors known to be related to an increased risk of sudden cardiac death, the only clinical practice currently used to predict such an event is the measurement of left ventricular ejection fraction. Use of this measurement for stratifying risk is based on the results of randomized trials of implantable defibrillators, which have shown a mortality benefit for patients with depressed left ventricular ejection fraction $(<35 \%)$, especially after a myocardial infarction ( $>40 \mathrm{~d}$ after event). ${ }^{3}$

Although left ventricular ejection fraction stratifies risk of sudden cardiac arrest for a specific subgroup of patients, it is an inadequate predictor of overall incidence because most randomized trials have focused on patients at high risk (i.e., patients who account for a low cumulative number of events). ${ }^{3}$ Therefore, the results of these studies cannot be accurately applied to the general population and have probably not had a substantial effect on the overall incidence of sudden cardiac arrest, as seen in statistics from the United States, ${ }^{4}$ despite the widespread use of such devices.

Traditional coronary risk factors, such as high cholesterol and hypertension, do not specifically identify those patients at high risk for sudden cardiac arrest. Studies involving samples drawn from the general population have shown that certain clinical and demographic variables are associated with risk of sudden cardiac arrest, such as male sex, exercise capacity, vital capacity, heart rate, having diabetes, obesity and smoking. ${ }^{5}$ Some electrocardiographic features, such as an early repolarization pattern in the inferior leads of a standard 12-lead electrocardiogram, have also recently been shown to predict the occurrence of sudden cardiac death in the general population. ${ }^{6}$ Factors such as obesity, diabetes, exercise habits and smoking could be mitigated using widespread population-based preventive strate- 
gies, which could help to reduce the overall incidence of sudden cardiac arrest. Other variables, such as electrocardiographic abnormalities, do not currently lend themselves to large-scale preventive strategies.

Ecologic studies are epidemiologic evaluations in which the units of analysis are populations, or groups of people, rather than individual people. ${ }^{7}$ Examples of these studies are evaluations of differences in care across different health care systems and studies of differences in outcomes for people living in different geographic regions or countries. Such studies are attracting the increased attention of policy-makers, managers of health care systems, clinicians and the public.

Ecologic studies that focus on disparities in socioeconomic status have shown that lower status is associated with poorer outcomes. This association has usually been attributed to differences in health behaviour ${ }^{8}$ or to fewer admissions of patients with low socioeconomic status to advanced medical care. ${ }^{9}$ Reinier and colleagues report that the incidence of sudden cardiac arrest is higher in poorer neighbourhoods in North American metropolitan areas. ${ }^{2}$ These results are not surprising - cardiovascular and cardiac mortality have been shown to be higher in populations with lower socioeconomic status, ${ }^{8,9}$ and approximately one half of cardiac deaths are sudden. In fact, almost all adverse outcomes, including mortality due to cancer, ${ }^{10}$ are higher in groups with the lowest socioeconomic status. Although their results may not be novel, Reinier and colleagues show in a subgroup analysis that the effect of disparate socioeconomic status on sudden cardiac arrest was most prominent in people under 65 years of age. These results thus merit further consideration in the development of strategies to reduce premature mortality in groups with low socioeconomic status.

Reinier and colleagues imply that population-based efforts to reduce cardiovascular risk factors and to treat unrecognized cardiovascular diseases in lower-income communities may reduce the incidence of premature, sudden, cardiac arrest, an idea that is well supported by the available information. Placement of automated external defibrillators in lower income communities, as suggested by the Reinier and colleagues, is not as well supported, as evidence of the effectiveness of this strategy is not yet available. The prevalence of fatal arrhythmias that could have been treated with an external defibrillator among the participants of this study was unknown, thus preventing us from drawing any conclusions as to the potential benefits of automated external defibrillators in this context. However, this study should inform the decisions of politicians and managers of health care systems as they institute health care reforms, recognizing that sudden cardiac arrest is the single most common cause of death in western societies.'

\section{References}

1. Huikuri HV, Castellanos A, Myerburg RJ. Sudden death due to cardiac arrhythmias. $N$ Engl J Med 2001;345:1473-82.

2. Reinier K, Thomas E, Andrusiek DL, et al. Socioeconomic status and incidence of sudden cardiac arrest.CMAJ 2011;183:1705-12.

3. Huikuri HV, Mäkikallio TH, Raatikainen MJ, et al. Prediction of sudden cardiac death. Appraisal of the studies and methods assessing the risk of sudden arrhythmic death. Circulation 2003;108:110-5.

4. Zheng ZJ, Croft JB, Giles WH, et al. Sudden cardiac death in the United States. Circulation 2001;104:2158-63.

5. Kannel WB, Schatzkin A. Sudden death: lessons from subsets in population studies. J Am Coll Cardiol 1985;5(Suppl):141B-9B

6. Tikkanen JT, Anttonen O, Junttila MJ, et al. Longterm outcome in patients with early repolarization patterns on electrocardiogram. $N$ Engl J Med 2009; 361:2529-37.

7. Tu JV, Ko DT. Ecological studies and cardiovascular outcomes research. Circulation 2008;118: 2588-93.

8. Stringhini S, Sabia S, Shipley M, et al. Association of socioeconomic position with health behaviors and mortality. JAMA 2010;303:1159-66.

9. Alter DA, Naylor CD, Austin P, et al. Effects of socioeconomic status on access to invasive cardiac procedures and on mortality after acute myocardial infarction. N Engl J Med 1999;341:1359-67.

10. Gorey KM, Holowaty EJ. Fehringer G, et al. An international comparison of cancer survival: relatively poor area of Toronto, Ontario and three US metropolitan areas. J Public Health Med 2000; 22:343-8.

Affiliation: Heikki V. Huikuri is with the Department of Internal Medicine, Institute of Clinical Medicine, University of Oulu, Oulu, Finland. 